\title{
Results from a PI-RADS-based MRI-directed diagnostic pathway for biopsy-naive patients in a non-university hospital
}

\author{
Jeroen S. Reijnen ${ }^{1,2}\left(\mathbb{D} \cdot\right.$ Jon B. Marthinsen ${ }^{1} \cdot$ Alf O. Tysland ${ }^{3} \cdot$ Christoph Müller $^{4}\left(\right.$ C) Irina Schönhardt $^{5}$. \\ Erlend Andersen ${ }^{1} \cdot$ Therese Seierstad $^{6}\left(\right.$ ID $\cdot$ Knut H. Hole ${ }^{2,6} \odot$
}

Received: 28 April 2021 / Revised: 11 August 2021 / Accepted: 11 August 2021 / Published online: 20 August 2021

(c) The Author(s) 2021

\begin{abstract}
Purpose To assess the safety and performance of a MRI-directed diagnostic pathway for patients with first-time suspicion of prostate cancer in a non-university hospital.

Methods Between May 2017 and December 2018 all biopsy-naive patients examined in our hospital followed a MRIdirected diagnostic work-up algorithm based on PI-RADS score. In short, PI-RADS 1-2 was generally not biopsied and PI-RADS 3-5 was reviewed by a multidisciplinary team. Patients with PI-RADS 4-5 were all referred to biopsy, either transrectal ultrasound-guided biopsy or MRI in-bore biopsy for small tumors and for sites difficult to access. PI-RADS scores were compared to the histopathology from biopsies and surgical specimens for patients who had prostatectomy. Non-biopsied patients were referred to a safety net monitoring regimen.

Results Two hundred and ninety-eight men were enrolled. 97 (33\%) had PI-RADS 1-2, 44 (15\%) had PI-RADS 3, and 157 (53\%) had PI-RADS 4-5. 116 (39\%) of the patients avoided biopsy. None of these were diagnosed with significant cancer within 2-3.5 years of safety net monitoring. Almost all high ISUP grade groups $(\geq 3)$ were in the PI-RADS 4-5 category (98\%). Prostatectomy specimens and systematic biopsies from MRI-negative areas indicated that very few clinically significant cancers were missed by the MRI-directed diagnostic pathway.

Conclusion Our findings add to evidence that a MRI-directed diagnostic pathway can be safely established in a non-university hospital. The pathway reduced the number of biopsies and reliably detected the site of the most aggressive cancers.
\end{abstract}

\section{Graphic abstract}

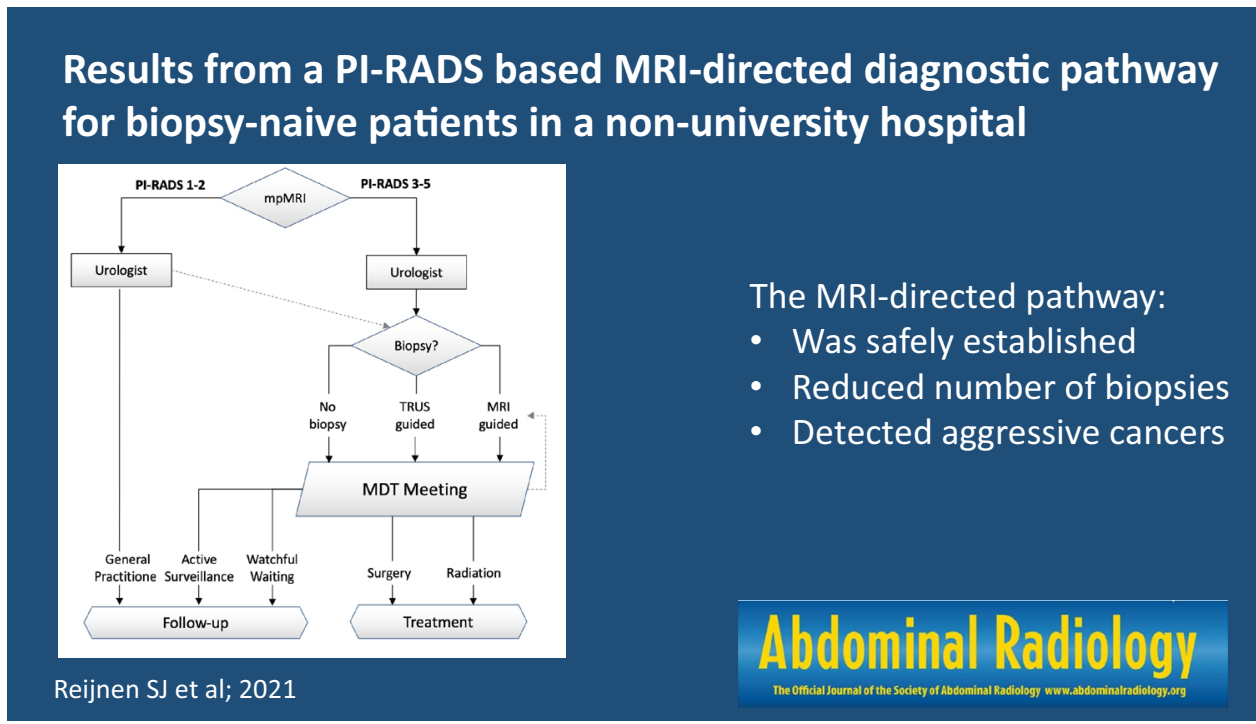

Extended author information available on the last page of the article 
Keywords Prostate neoplasms $\cdot$ Magnetic resonance imaging $\cdot$ Biopsy $\cdot$ Patient care team $\cdot$ Algorithms

\section{Introduction}

Magnetic resonance imaging (MRI) is increasingly used in the diagnostic work-up of biopsy-naive patients with suspected prostate cancer. The ability of MRI to detect and localize clinically significant prostate cancer has been established [1-6] and MRI-directed biopsy strategies have been shown to benefit biopsy-naive patients [7-10]. A recent Cochrane review concluded that MRI-directed diagnostic work-up increased the detection of clinically significant prostate cancer and reduced the detection of insignificant prostate cancer compared to systematic biopsy [11]. On the basis of this body of evidence, the Prostate ImagingReporting and Data System (PI-RADS) committee recently proposed the PI-RADS MRI-directed biopsy pathway [12].

PI-RADS is both observer- and experience-dependent [13-16] and the performance of a MRI-directed diagnostic pathway also relies on adequate biopsy targeting [10, 17, 18]. Thus, to achieve widespread adoption of MRI-directed diagnostic pathways without routine systematic biopsies, a large and varied body of knowledge is needed. Most of the data collected so far originate from studies with multicenter design, with the majority of data collected from academic, tertiary referral centers $[5,7,18]$. Data from non-university hospitals are highly warranted [11, 12, 16, 19].

Based on the results of the PROMIS trial [2], we introduced in 2017 a MRI-directed diagnostic pathway for biopsy-naive patients at our non-university hospital. Our pathway is similar to the pathway proposed by the PIRADS-steering committee in 2019 [12]. The purpose of this study was to report the performance of our MRI-directed diagnostic pathway for patients with first-time suspicion of prostate cancer.

\section{Materials and methods}

\section{Study cohort and patient workflow}

Almost all patients with suspected prostate cancer within the local county are referred to the prostate cancer referral pathway at our hospital. The referral population is 187.000. All referrals to our hospital for first-time suspicion of prostate cancer were assessed by an urologist who initiated the pathway. After inclusion into the prostate cancer referral pathway, all patients had MRI prior to biopsy. A flow-chart summarizing the MRI-directed pathway is shown in Fig. 1. The data from these patients were prospectively entered into the Institutional Prostate Cancer Quality Registry.
This retrospective single-center study of prospectively collected clinical routine data was approved by the Institutional Review Board that waived the need for informed consent. From May 2017 until December 2018 data from a total of 298 patients were enrolled. The mean age was 61 years (standard deviation 7.6, range 31-75) and the mean prostatespecific antigen (PSA) was $5.9 \mathrm{ng} / \mathrm{ml}$ (standard deviation 7.2 , range $0.7-40$ ).

\section{Multiparametric MRI}

All patients were examined using a $3 \mathrm{~T}$ Siemens MAGNETOM Skyra MRI scanner and phased-array coils. Preparation of the patient included a cleansing enema (toilax $10 \mathrm{mg} / 5 \mathrm{ml}$ bisakodyl, Orion Corporation) to void the rectum and intravenous administration of $2 \mathrm{ml}$ Buscopan (Boehringer Ingelheim) and intramuscular injection of $1 \mathrm{mg}$ Glucagon (Novo Nordisk A/S) to reduce spasmodic activity. The MRI protocol included morphological T1- and T2-weighted images as well as diffusion-weighted images and dynamic contrast-enhanced (DCE) images. The MRI sequences and acquisition parameters are summarized in Online Resource 1 . The image quality complies with the technical requirements from PI-RADS v2 and 2.1 [16, 20, 21]. DCE imaging was performed after $i . v$. injection of gadoterate meglumine (DOTAREM, Guerbet LLC, ) at a dose of $0.1 \mathrm{mmol} / \mathrm{kg}$ body weight at a rate of $2 \mathrm{ml} / \mathrm{s}$ followed by a $20 \mathrm{ml}$ saline flush. All MRI examinations were independently interpreted by one of two experienced radiologists (JSR, JBM) with 10 and 5 years of experience with prostate MRI, respectively. The results from the clinical exam by the general practitioner and serum PSA level were known to the readers when interpreting the images. For each patient, up to three lesions were assigned a score according to the PIRADS v2 [20]. The location and extent of the lesions were drawn in the PI-RADS report template (Fig. 2).

\section{Prostate biopsy}

Based on clinical information, PI-RADS score, and a template drawing of tumor localization and extent, the urologist decided the next step and the need for biopsy. For patients with PI-RADS 1-2 no biopsy was performed unless risk factors were present (PSA metrics, digital rectal examination findings, family history, comorbidity, and life expectancy). Systematic transrectal ultrasound (TRUS) biopsies were obtained for patients with risk factors. For patients with PI-RADS 3 the decision on whether to biopsy was made by the multidisciplinary team (MDT) based on MRI and risk factors. Patients with PI-RADS 4-5 were all referred 
Fig. 1 Flow-chart of the MRIdirected pathway for patients with suspicion of prostate cancer referred to our hospital. TRUSGB transrectal ultrasoundguided biopsy, $S B$ systematic biopsies, $T B$ MRI-targeted biopsies with cognitive fusion, $M R I G B$ magnetic resonance imaging-guided biopsy, $M D T$ multidisciplinary team

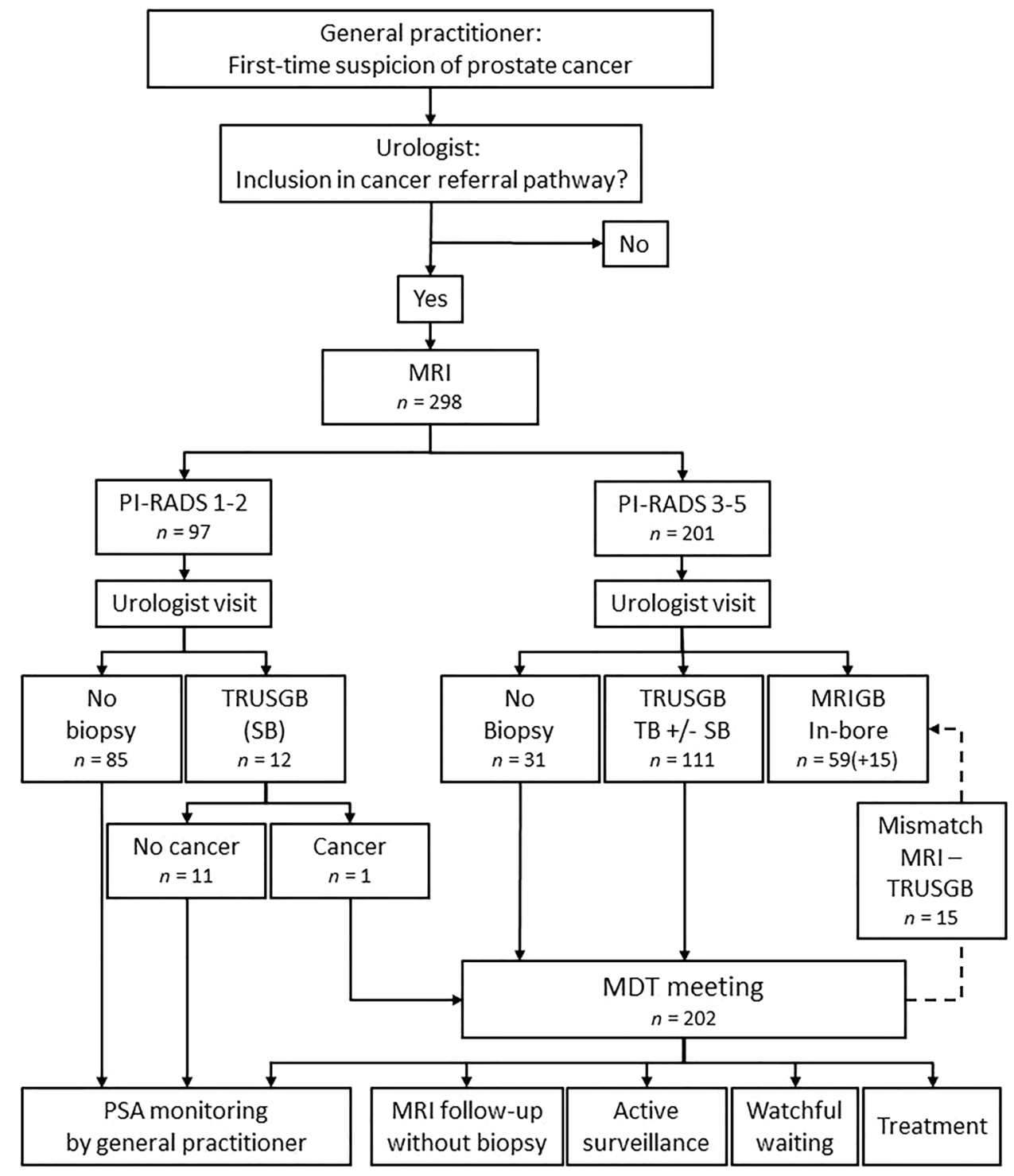

to biopsy, either TRUS biopsies or MRI in-bore biopsy for small tumors and for sites difficult to access. Patients with TRUS-guided biopsies had targeted biopsies and, in most cases, also 12-core systematic biopsies. In some patients with large tumors at MRI, systematic biopsies were omitted. The systematic biopsies were obtained according to a standardized template that allowed correlation to MRI findings. Targeting was performed using cognitive fusion based on the template drawing (Fig. 2).

Transrectal in-bore MRI-guided biopsies were performed by one of the two radiologists or in collaboration, using the same scanner as for diagnostic MRI. Robotic assistance (Remote Controlled Manipulator, Soteria Medical) was used for steering the needle guide (Invivo). Two to four biopsies per target lesion were obtained with an $18 \mathrm{G}$ biopsy needle (Tru-Core II, Argon Medical Devices). The biopsy specimens were fixed in $10 \%$ buffered formalin, embedded in paraffin, and stained with hematoxylin and eosin (HE), according to our standard hospital protocol. An experienced uropathologist (IS) evaluated the biopsies and assigned a grade group according to the International Society of Urological Pathology (ISUP) criteria [22]. Clinically significant cancer was defined as Gleason score of at least $3+4$ (ISUP grade group $\geq 2)[12,22]$.

\section{Multidisciplinary team and safety net}

The prostate MDT consisted of urologists, radiologists, oncologists, and a pathologist and met weekly. All PIRADS 3-5 and PI-RADS 1-2 with positive biopsy were discussed (Fig. 1). Further, the urologist had the option to consult the MDT when in doubt on whether to biopsy a patient with PI-RADS 1-2. If MDT suspected that the biopsy findings were not representative, i.e., mismatch 
Fig. 2 Example of the template drawing of the location and extent of the prostate cancer as depicted by MRI
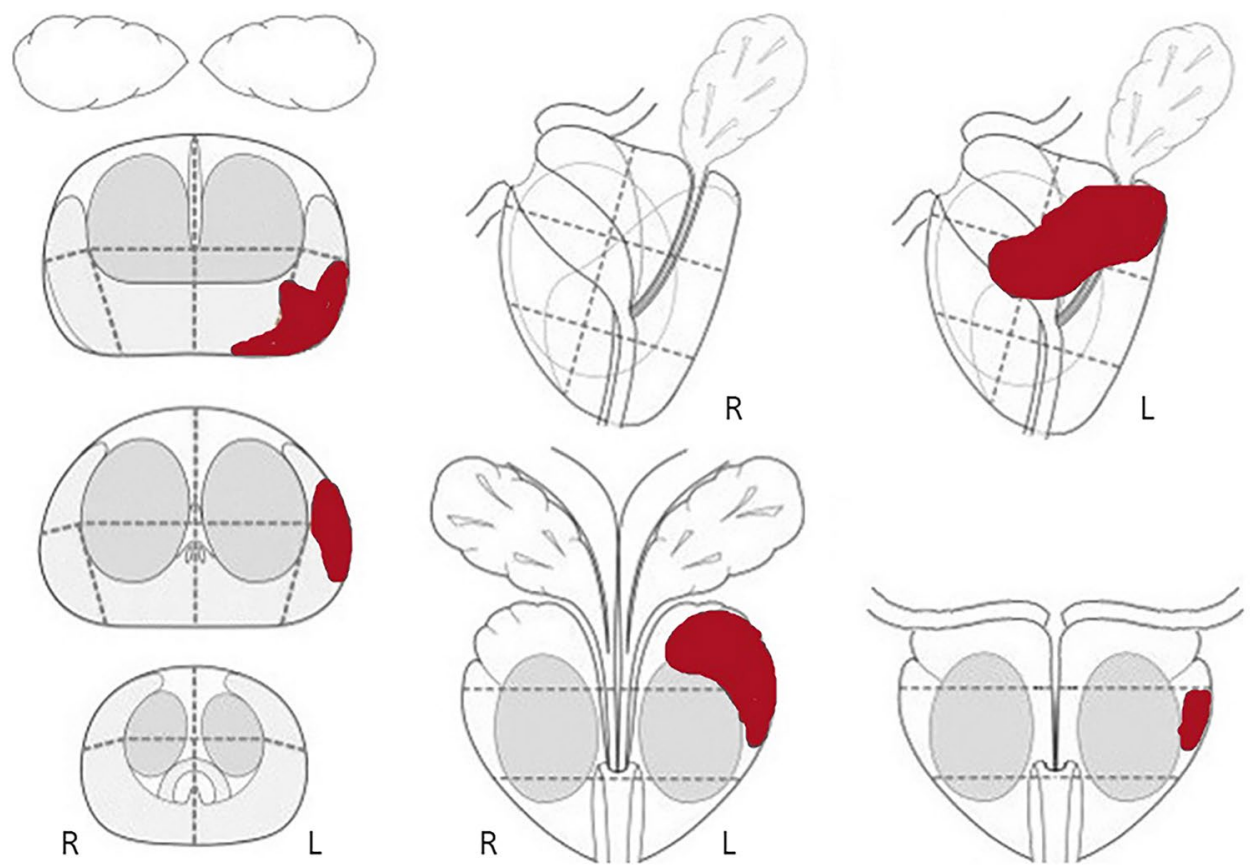

between findings at MRI and the results from TRUS biopsies, patients were referred to additional targeted in-bore MRI biopsy. For patients with detected prostate cancer the MDT decided whether the patient should undergo treatment or be subjected to follow-up (active surveillance or watchful waiting).

A safety net was established for non-biopsied patients. Non-biopsied PI-RADS 1-2 patients were referred to followup by the GP with instruction to contact the urologist at our hospital if the PSA metrics increased above a threshold value. Non-biopsied patients with PI-RADS 3 were followed up by PSA measurements mainly at six-month intervals at the hospital (12 of 21) or by the GP ( 9 of 21). Five of the patients followed at the hospital had a new MRI and three were biopsied later in the follow-up period without clinically significant cancer. It is important to point out that the public health care system in our region is organized so that all patients will be referred back to our hospital exclusively. This, we consider an important part of the oncologic safety.

\section{Data analyses}

Descriptive statistics are used to present our experiences with the MRI-directed prostate pathway. PI-RADS scores, per patient and per lesion, were compared to the histopathology from biopsy and surgical specimens for patients who had prostatectomy. PI-RADS scores 1-2 and scores 4-5 were grouped for the data analysis. For the analyses per patient, the highest overall PI-RADS score for each patient was used.

\section{Results}

Figure 3 shows representative MR images of one of the patients. Of the 298 patients, 97 (33\%) had PI-RADS 1-2, 44 (15\%) had PI-RADS 3, and 157 (53\%) had PI-RADS 4-5. 116 (39\%) were not biopsied but referred to PSA monitoring or follow-up with MRI. Of these 116 non-biopsied patients, 85 (73\%) had PI-RADS 1-2, 21 (19\%) had PI-RADS 3, and 10 (9\%) had PI-RADS 4-5. The reasons for not performing biopsy of the 10 patients with PI-RADS 4-5 were inflammation risk $(n=1)$, patient choice $(n=6)$, differential diagnosis of inflammation and rapid PSA decrease after MRI $(n=1)$, and MRI indicated a small, low-grade tumor combined with high age $(n=1)$ or comorbidity $(n=1)$.

Of the 298 patients, 108 had TRUS-guided biopsy and 74 had in-bore MRI biopsy, 15 of these after TRUS-guided biopsy (Fig. 1). In these 15 patients, 8 tumors were upgraded from ISUP 1 to 2, two from benign to ISUP 2, and five were unchanged.

The histopathological findings per patient as function of PI-RADS are summarized in Fig. 4. Prostate cancer was detected in 139 (46\%) patients. Of these, $30(22 \%)$ had ISUP 1, 65 (46\%) had ISUP 2, 21 (15\%) had ISUP 3, and $23(16 \%)$ had ISUP 4-5. No cancer was found in $43(24 \%)$ of the 182 biopsied patients. No clinically significant cancer was found in the PI-RADS 1-2 category and in only three of the 44 patients (7\%) with PI-RADS 3. 42 of 43 high ISUP grade groups $(\geq 3)$ were in the PI-RADS $4-5$ category. 88 of 95 lower ISUP grade groups $(\leq 2)$ were also in PI-RADS 4-5. No non-biopsied patients or PI-RADS 4-5 patients with benign biopsies were referred back to our hospital 

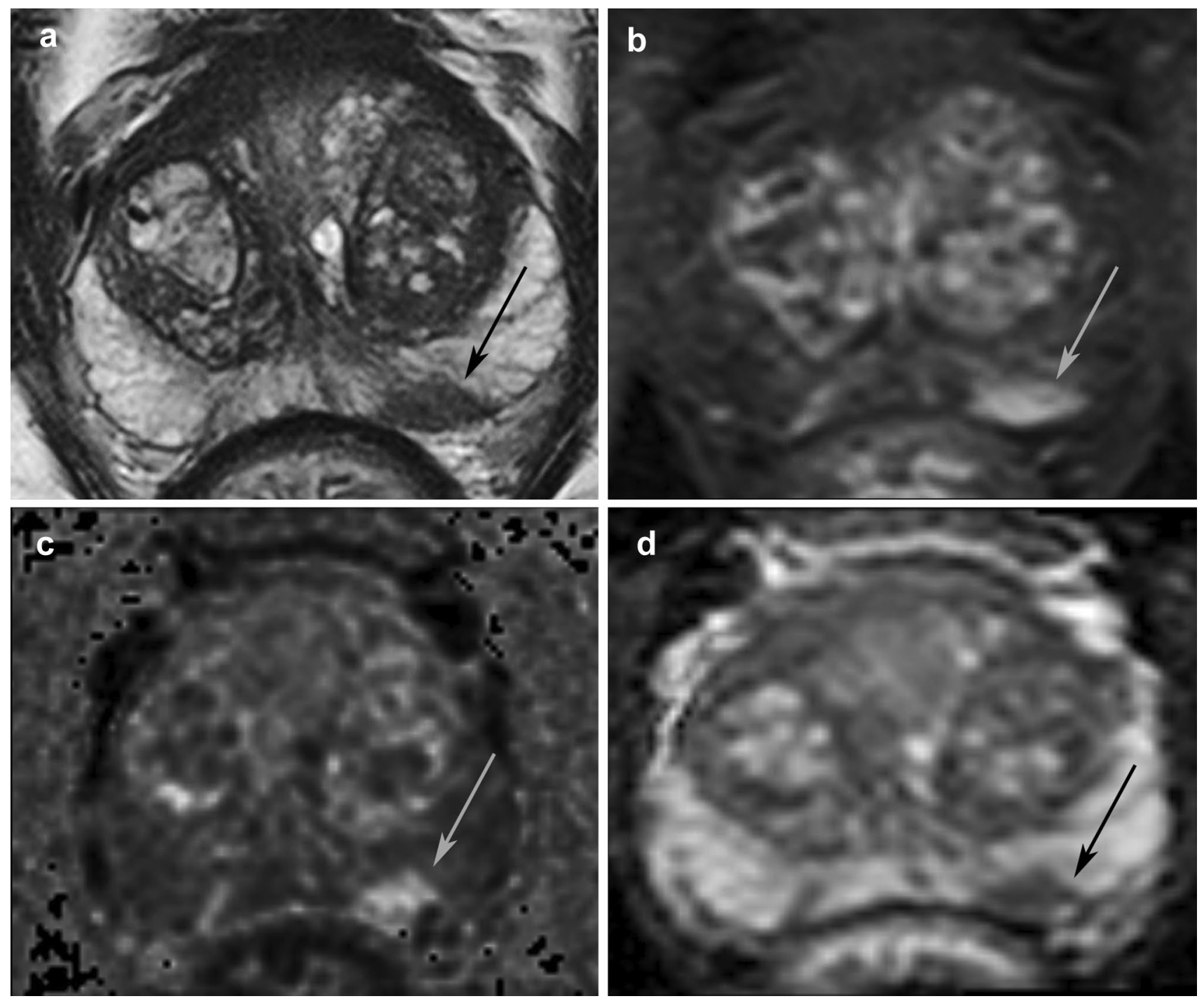

Fig. 3 Representative MRI images used in our MRI-directed diagnostic pathway for prostate cancer. 60-year-old man with an ISUP grade group 2 cancer posterolaterally in the peripheral zone left

side (arrow). a Transversal T2W, b early-phase dynamic contrastenhanced T1W DIXON water only, c zoomed DWI with calculated b1400, and $\mathbf{d}$ ADC from b0 to b800

and diagnosed with clinically significant cancer during the 2.0-3.5-year-follow-up time.

Some patients had multiple lesions, in total 445 lesions were PI-RADS classified. The results per lesion are summarized in Online Resource 2.

All except three of the 111 PI-RADS 3-5 patients with TRUS-guided biopsies also had systematic biopsies covering MRI-negative regions. Eleven of these patients had positive biopsies from MRI-negative regions. All except one were lower than or equal in ISUP grade group than from the MRI-positive areas. One patient had a large PI-RADS 5 tumor harboring ISUP 4 on one side, with ISUP 4 and 5 in systematic biopsies on the other side.

Of the 298 patients, 38 had prostatectomy. Five were upgraded from the biopsies to the resected specimen: four

from ISUP 2 to ISUP 3 and one from ISUP 1 to ISUP 3 (Online Resource 3).

\section{Discussion}

In June 2019 the PI-RADS-steering committee advocated to implement a MRI-directed biopsy pathway enabling key diagnostic benefits to men with suspected prostate cancer [12]: that is to (a) reduce the number of patients who need prostate biopsy, (b) reduce the detection of clinically insignificant cancer, (c) increase detection of clinically significant cancers, and (d) improve risk stratification. Our two years of experience with a MRI-directed diagnostic pathway show that implementation in clinical routine in a non-university 
Fig. 4 PI-RADS and biopsy findings for the 298 patients who were referred to our MRIdirected diagnostic pathway. TRUS transrectal ultrasound, ISUP International Society of Urological Pathology

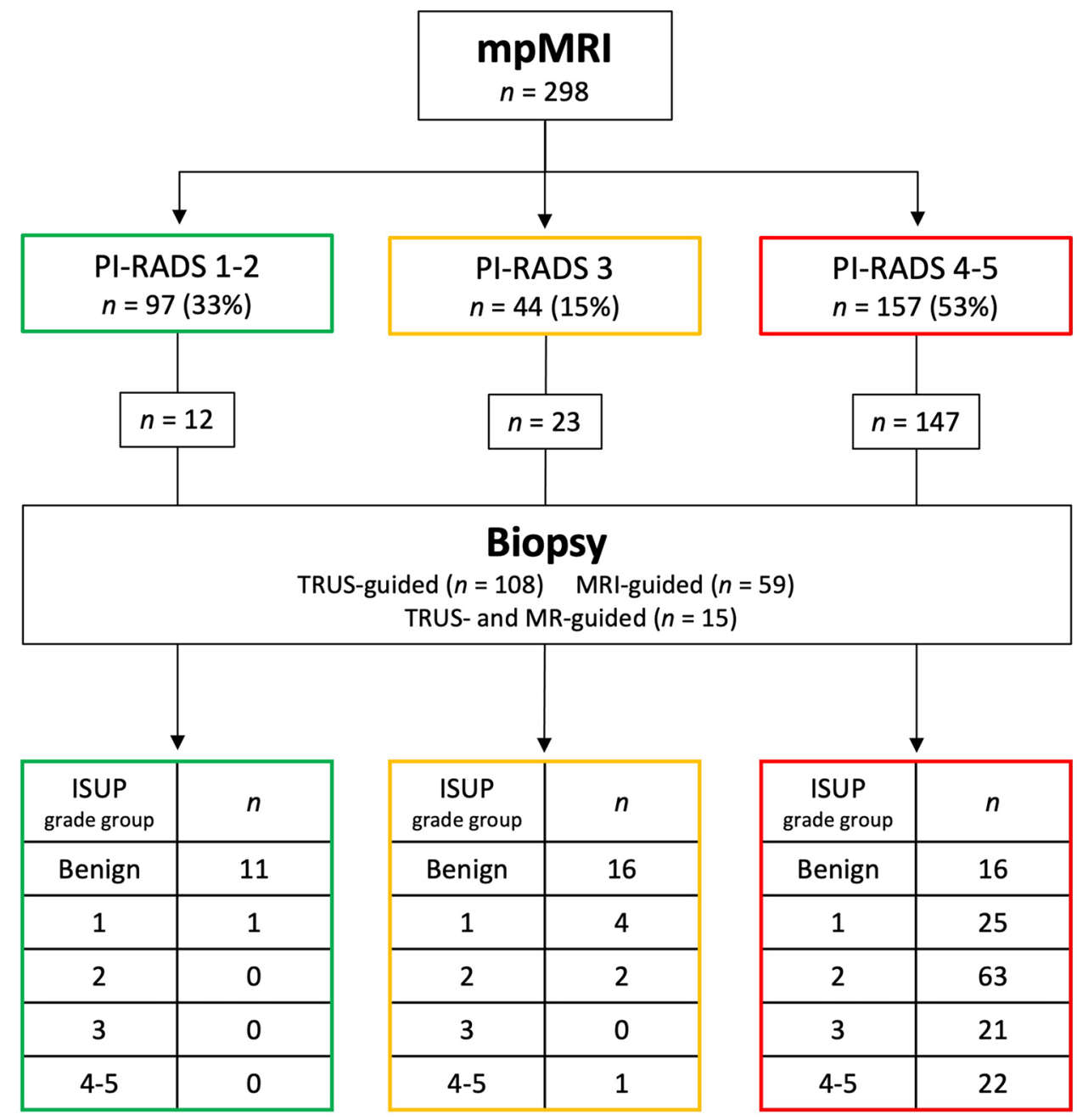

hospital is feasible and that the anticipated benefits can be obtained.

About $40 \%$ of our patients did not undergo biopsy and the majority of them had PI-RADS 1-2. This is in line with other 'real-world' results reported from the IMRIE study and by Sokhi et al. [23, 24]. Without template biopsies it is not possible to rule out that significant cancers have been overlooked. However, we had findings indicating that this is unlikely: of the 12 patients with PI-RADS 1-2 who underwent biopsy, none was diagnosed with clinically significant cancer (one had ISUP 1). Although limited follow-up time (2-3.5 years), none of the patients have been referred back to our hospital and diagnosed with clinically significant cancer.

Almost half of the PI-RADS 3 patients did not undergo immediate biopsy, an optional strategy in the algorithm proposed by the PI-RADS-steering committee. Of the biopsied PI-RADS 3 patients, two had ISUP 2 and one had ISUP 3 . The number of PI-RADS 3 patients diagnosed with clinically significant cancer (7\%) was lower than in other studies $(12-31 \%)[5,7,10]$. A possible explanation is that we used high-resolution DWI and DCE images making small lesions appear more discrete and with higher contrast between the lesion and the background. This could migrate some PIRADS 3 lesions to PI-RADS 4.

The detection of ISUP 1 was $10 \%$ in our entire cohort. This is comparable to the results from MRI-directed strategies in multicenter trials and significantly lower than for the strategy using systematic biopsies in these trails [7, 10, 18]. In the MRItargeted biopsy group we found 17\% ISUP 1, also comparable to the results from these trials. Apparently, high-quality PI-RADS multiparametric MRI detects insignificant disease despite its aim not to do so [8]. One strategy to reduce this could be to lower the sensitivity of PI-RADS MRI, either by altering the interpretation criteria (PI-RADS) or reduce the sensitivity of the imaging. However, such adjustments can be expected to negatively impact detection of clinically significant cancer. An alternative strategy could be to incorporate parameters into PIRADS and MRI that reflect tumor aggressiveness [25].

Our findings are similar to prospective multicenter trials documenting that a MRI-directed pathway in biopsy-naive patients can detect clinically significant cancer with high sensitivity $[2,7,10,18]$. But is it safe to omit systematic 
biopsies? It has been shown that MRI misses some ISUP 2 $[2,26]$ and that systematic biopsies detect some of those [5, 17, 18]. The ultimate reference standard to detect upgrading is prostatectomy. In our cohort 38 patients underwent surgery and in five patients the histopathology was upgraded, four from ISUP 2 to 3 and one from ISUP 1 to 3 . We also tried to detect missed cancers by looking for positive systematic biopsies from MRI-negative regions. We found 11 patients, and all except one were lower or equal in ISUP than from their MRI-positive regions.

The main limitation of our study was the lack of systematic template-based biopsies in all patients. The study was not designed to measure the accuracy of MRI, but to gain evidence of the safety of a MRI-directed diagnostic pathway implemented in 'real-world' clinical care. Second, the follow-up time of non-biopsied patients is limited. However, a longer follow-up time has a trade-off because it would be difficult to distinguish overlooked cancers from de novo transformed cancers. Third, the generalizability to other hospitals depends not only of the algorithm itself but also on the quality of all its steps. Especially the imaging protocol and patient preparations for the MRI may influence the generalizability as it varies widely in the literature and between institutions $[16,21,27]$. The strength of our study is that we attempted to optimize all steps through high image quality, experienced radiologists, high-precision biopsy targeting when required, and MDT composed of all involved disciplines: radiologist, urologist, oncologist, and pathologist.

In conclusion, our findings add to evidence that a MRIdirected diagnostic pathway can be safely established in a non-university hospital and delivers improved risk stratification, as advocated by the PI-RADS-steering committee [12]. Prostate cancer of uncertain clinical significance is still diagnosed.

Supplementary Information The online version contains supplementary material available at https://doi.org/10.1007/s00261-021-03249-8.

Author contributions All authors made substantial contributions to the conception or design of the work, or the acquisition, analysis, or interpretation of data. JSR, KHH, and TS drafted the work and JBM, AOT, CM, IS, and EA revised it critically for important intellectual content. All authors approved the version to be published and agreed to be accountable for all aspects of the work.

Funding Open access funding provided by University of Oslo (incl Oslo University Hospital). No funds, grants, or other support were received.

\section{Declarations}

Conflict of interest The authors have no relevant financial or non-financial interests to disclose.

Ethical approval The Institutional Review Board waived the need for informed consent.
Open Access This article is licensed under a Creative Commons Attribution 4.0 International License, which permits use, sharing, adaptation, distribution and reproduction in any medium or format, as long as you give appropriate credit to the original author(s) and the source, provide a link to the Creative Commons licence, and indicate if changes were made. The images or other third party material in this article are included in the article's Creative Commons licence, unless indicated otherwise in a credit line to the material. If material is not included in the article's Creative Commons licence and your intended use is not permitted by statutory regulation or exceeds the permitted use, you will need to obtain permission directly from the copyright holder. To view a copy of this licence, visit http://creativecommons.org/licenses/by/4.0/.

\section{References}

1. Futterer JJ, Briganti A, De Visschere P, Emberton M, Giannarini G, Kirkham A, Taneja SS, et al (2015) Can Clinically Significant Prostate Cancer Be Detected with Multiparametric Magnetic Resonance Imaging? A Systematic Review of the Literature. Eur Urol 68:1045-1053. https://doi.org/10.1016/j.eururo.2015.01.013

2. Ahmed HU, El-Shater Bosaily A, Brown LC, Gabe R, Kaplan R, Parmar MK, Collaco-Moraes Y, et al (2017) Diagnostic accuracy of multi-parametric MRI and TRUS biopsy in prostate cancer (PROMIS): a paired validating confirmatory study. Lancet 389:815-822. https://doi.org/10.1016/S0140-6736(16)32401-1

3. Woo S, Suh CH, Kim SY, Cho JY, Kim SH (2017) Diagnostic Performance of Prostate Imaging Reporting and Data System Version 2 for Detection of Prostate Cancer: A Systematic Review and Diagnostic Meta-analysis. Eur Urol 72:177-188. https://doi.org/ 10.1016/j.eururo.2017.01.042

4. Zhang L, Tang M, Chen S, Lei X, Zhang X, Huan Y (2017) A meta-analysis of use of Prostate Imaging Reporting and Data System Version 2 (PI-RADS V2) with multiparametric MR imaging for the detection of prostate cancer. Eur Radiol 27:5204-5214. https://doi.org/10.1007/s00330-017-4843-7

5. Hansen NL, Barrett T, Kesch C, Pepdjonovic L, Bonekamp D, O'Sullivan R, Distler F, et al (2018) Multicentre evaluation of magnetic resonance imaging supported transperineal prostate biopsy in biopsy-naive men with suspicion of prostate cancer. BJU Int 122:40-49. https://doi.org/10.1111/bju.14049

6. Moldovan PC, Van den Broeck T, Sylvester R, Marconi L, Bellmunt J, van den Bergh RCN, Bolla M, Briers E, et al (2017) What Is the Negative Predictive Value of Multiparametric Magnetic Resonance Imaging in Excluding Prostate Cancer at Biopsy? A Systematic Review and Meta-analysis from the European Association of Urology Prostate Cancer Guidelines Panel. Eur Urol 72:250-266. https://doi.org/10.1016/j.eururo.2017.02.026

7. Kasivisvanathan V, Rannikko AS, Borghi M, Panebianco V, Mynderse LA, Vaarala MH, Briganti A, Budaus L, et al (2018) MRI-Targeted or Standard Biopsy for Prostate-Cancer Diagnosis. N Engl J Med 378:1767-1777. https://doi.org/10.1056/NEJMo a1801993

8. Padhani AR, Weinreb J, Rosenkrantz AB, Villeirs G, Turkbey B, Barentsz J (2019) Prostate Imaging-Reporting and Data System Steering Committee: PI-RADS v2 Status Update and Future Directions. Eur Urol 75:385-396. https://doi.org/10.1016/j.eururo. 2018.05.035

9. Panebianco V, Barchetti F, Sciarra A, Ciardi A, Indino EL, Papalia R, Gallucci M, et al (2015) Multiparametric magnetic resonance imaging vs. standard care in men being evaluated for prostate cancer: a randomized study. Urol Oncol 33:17 e11-17 e17. https:// doi.org/10.1016/j.urolonc.2014.09.013

10. van der Leest $M$, Cornel $E$, Israel $B$, Hendriks R, Padhani AR, Hoogenboom M, Zamecnik P, et al (2019) Head-to-head 
Comparison of Transrectal Ultrasound-guided Prostate Biopsy Versus Multiparametric Prostate Resonance Imaging with Subsequent Magnetic Resonance-guided Biopsy in Biopsy-naive Men with Elevated Prostate-specific Antigen: A Large Prospective Multicenter Clinical Study. Eur Urol 75:570-578. https://doi. org/10.1016/j.eururo.2018.11.023

11. Drost FH, Osses DF, Nieboer D, Steyerberg EW, Bangma CH, Roobol MJ, Schoots IG (2019) Prostate MRI, with or without MRI-targeted biopsy, and systematic biopsy for detecting prostate cancer. Cochrane Database Syst Rev 4:CD012663. https://doi.org/ 10.1002/14651858.CD012663.pub2

12. Padhani AR, Barentsz J, Villeirs G, Rosenkrantz AB, Margolis DJ, Turkbey B, Thoeny HC, et al (2019) PI-RADS Steering Committee: The PI-RADS Multiparametric MRI and MRI-directed Biopsy Pathway. Radiology 292:464-474. https://doi.org/10.1148/ radiol.2019182946

13. Greer MD, Brown AM, Shih JH, Summers RM, Marko J, Law YM, Sankineni S, et al (2017) Accuracy and agreement of PIRADSv2 for prostate cancer mpMRI: A multireader study. J Magn Reson Imaging 45:579-585. https://doi.org/10.1002/jmri. 25372

14. Rosenkrantz AB, Ginocchio LA, Cornfeld D, Froemming AT, Gupta RT, Turkbey B, Westphalen AC, et al (2016) Interobserver Reproducibility of the PI-RADS Version 2 Lexicon: A Multicenter Study of Six Experienced Prostate Radiologists. Radiology 280:793-804. https://doi.org/10.1148/radiol.2016152542

15. Rosenkrantz AB, Oto A, Turkbey B, Westphalen AC (2016) Prostate Imaging Reporting and Data System (PI-RADS), Version 2: A Critical Look. AJR Am J Roentgenol 206:1179-1183. https:// doi.org/10.2214/AJR.15.15765

16. Turkbey B, Rosenkrantz AB, Haider MA, Padhani AR, Villeirs G, Macura KJ, Tempany CM, et al (2019) Prostate Imaging Reporting and Data System Version 2.1: 2019 Update of Prostate Imaging Reporting and Data System Version 2. Eur Urol 76:340-351. https://doi.org/10.1016/j.eururo.2019.02.033

17. Muthigi A, George AK, Sidana A, Kongnyuy M, Simon R, Moreno V, Merino MJ, et al (2017) Missing the Mark: Prostate Cancer Upgrading by Systematic Biopsy over Magnetic Resonance Imaging/Transrectal Ultrasound Fusion Biopsy. J Urol 197:327-334. https://doi.org/10.1016/j.juro.2016.08.097

18. Rouviere O, Puech P, Renard-Penna R, Claudon M, Roy C, MegeLechevallier F, Decaussin-Petrucci M, et al (2019) Use of prostate systematic and targeted biopsy on the basis of multiparametric MRI in biopsy-naive patients (MRI-FIRST): a prospective, multicentre, paired diagnostic study. Lancet Oncol 20:100-109. https:// doi.org/10.1016/S1470-2045(18)30569-2
19. Lebastchi AH, Pinto PA (2019) The role of multiparametric MRI in biopsy-naive prostate cancer. Nat Rev Urol 16:276-277. https:// doi.org/10.1038/s41585-019-0173-7

20. Weinreb JC, Barentsz JO, Choyke PL, Cornud F, Haider MA, Macura KJ, Margolis D, et al (2016) PI-RADS Prostate Imaging - Reporting and Data System: 2015, Version 2. Eur Urol 69:16-40. https://doi.org/10.1016/j.eururo.2015.08.052

21. de Rooij M, Israel B, Tummers M, Ahmed HU, Barrett T, Giganti F, Hamm B, et al (2020) ESUR/ESUI consensus statements on multi-parametric MRI for the detection of clinically significant prostate cancer: quality requirements for image acquisition, interpretation and radiologists' training. Eur Radiol 30:5404-5416. https://doi.org/10.1007/s00330-020-06929-z

22. Epstein JI, Egevad L, Amin MB, Delahunt B, Srigley JR, Humphrey PA, Grading C (2016) The 2014 International Society of Urological Pathology (ISUP) Consensus Conference on Gleason Grading of Prostatic Carcinoma: Definition of Grading Patterns and Proposal for a New Grading System. Am J Surg Pathol 40:244-252. https://doi.org/10.1097/PAS.0000000000000530

23. Sokhi HK, Padhani AR, Patel S, Pope A (2020) Diagnostic yields in patients with suspected prostate cancer undergoing MRI as the first-line investigation in routine practice. Clinical Radiology 75:950-956. https://doi.org/10.1016/j.crad.2020.08.011

24. Stonier T, Simson N, Shah T, Lobo N, Amer T, Lee S-M, Bass E, et al (2020) The 'Is mpMRI Enough' or IMRIE Study: A Multicentre Evaluation of Prebiopsy Multiparametric Magnetic Resonance Imaging Compared with Biopsy. European Urology Focus S2405-4569(20)30271-6 https://doi.org/10.1016/j.euf.2020.09. 012

25. Hompland T, Hole KH, Ragnum HB, Aarnes EK, Vlatkovic L, Lie AK, Patzke S, et al (2018) Combined MR Imaging of Oxygen Consumption and Supply Reveals Tumor Hypoxia and Aggressiveness in Prostate Cancer Patients. Cancer Res 78:4774-4785. https://doi.org/10.1158/0008-5472.CAN-17-3806

26. Borofsky S, George AK, Gaur S, Bernardo M, Greer MD, Mertan FV, Taffel M, et al (2018) What Are We Missing? False-Negative Cancers at Multiparametric MR Imaging of the Prostate. Radiology 286:186-195. https://doi.org/10.1148/radiol.2017152877

27. Giganti F, Kirkham A, Kasivisvanathan V, Papoutsaki MV, Punwani S, Emberton M, Moore CM, et al (2021) Understanding PI-QUAL for prostate MRI quality: a practical primer for radiologists. Insights Imaging 12:59. https://doi.org/10.1186/ s13244-021-00996-6

Publisher's Note Springer Nature remains neutral with regard to jurisdictional claims in published maps and institutional affiliations.

\section{Authors and Affiliations}

\section{Jeroen S. Reijnen ${ }^{1,2}\left(\mathbb{D} \cdot\right.$ Jon B. Marthinsen ${ }^{1} \cdot$ Alf O. Tysland ${ }^{3}$. Christoph Müller ${ }^{4}\left(\mathbb{D} \cdot\right.$ Irina Schönhardt $^{5}$. Erlend Andersen ${ }^{1} \cdot$ Therese Seierstad $^{6}\left({ }^{(0)} \cdot\right.$ Knut H. Hole $^{2,6}{ }^{10}$}

Therese Seierstad

therese@ radium.uio.no

1 Department of Radiology, Sørlandet Hospital, Kristiansand, Norway

2 Institute of Clinical Medicine, University of Oslo, Oslo, Norway

3 Department of Urology, Sørlandet Hospital, Kristiansand, Norway
4 Department for Cancer Treatment, Sørlandet Hospital, Kristiansand, Norway

5 Department of Pathology, Sørlandet Hospital, Kristiansand, Norway

6 Division of Radiology and Nuclear Medicine, Radiumhospitalet, Oslo University Hospital, P.o.box 4950, Nydalen, Oslo, Norway 